\title{
Frontal EEG asymmetry moderates the effects of stressful life events on internalizing symptoms in children at familial risk for depression
}

\author{
NESTOR L. LOPEZ-DURAN, ${ }^{a}$ ROBIN NUSSLOCK, ${ }^{\mathrm{b}}$ CHARLES GEORGE, ${ }^{\mathrm{c}}$ AND MARIA KOVACS ${ }^{\mathrm{d}}$ \\ ${ }^{a}$ Department of Psychology, University of Michigan-Ann Arbor, Ann Arbor, Michigan, USA \\ ${ }^{b}$ Department of Psychology, Northwestern University, Chicago, Illinois, USA \\ 'Department of Psychiatry, University of Pittsburgh Medical Center, Pittsburgh, Pennsylvania, USA \\ ${ }^{\mathrm{d} D e p a r t m e n t}$ of Psychiatry, University of Pittsburgh School of Medicine, Pittsburgh, Pennsylvania, USA
}

\begin{abstract}
This study examined whether frontal alpha electroencephalographic (EEG) asymmetry moderates the association between stressful life events and depressive symptoms in children at familial risk for depression. Participants included 135 children ages 6 to 13, whose mothers had either a history of depression or no history of major psychiatric conditions. Frontal EEG was recorded while participants watched emotion-eliciting films. Symptoms and stressful life events were obtained via the Child Behavior Check List and a clinical interview, respectively. High-risk children displayed greater relative right lateral frontal activation (F7/F8) than their low-risk peers during the films. For high-risk children, greater relative left lateral frontal activation moderated the association between stressful life events and internalizing symptoms. Specifically, greater relative left lateral frontal activation mitigated the effects of stress in at-risk children.
\end{abstract}

Descriptors: Child depression, EEG, Brain asymmetry, Life stressors

A number of cross-sectional and longitudinal studies indicate that acute stressful events as well as the accumulation of life stressors are associated with depressive symptoms and the onset of major depression in children and adolescents (Goodyer, Herbert, Tamplin, \& Altham, 2000; Goodyer, Kolvin, \& Gatzanis, 1985; Williamson et al., 1998). For over 20 years, researchers have explored why some people appear to be more predisposed to suffer from the effects of stress while others seem resilient, with most research focusing on the possible role of cognitive, personality, and genetic factors (e.g., Alloy et al., 2000; Caspi et al., 2003). One possible moderator of the effect of stress on depression, namely, affective style as indexed by frontal alpha electroencephalographic (EEG) asymmetry (see Davidson, 2004), has received less attention despite its role in modulating an individual's response to environmental stressors (e.g., Tomarken, Davidson, \& Henriques, 1990). The examination of the interplay between affective style and stressful life events is particularly important in the study of children at familial risk for depression, because these offspring are exposed to elevated levels of stressful life events (Goodman \& Gotlib, 1999) and display cognitive, affective, and physiological profiles that may place them at higher risk for a dysregulated response to negative

This study was supported by NIMH Program Project grant PO1 MH056193 to Dr. Kovacs. Dr. Nathan Fox was the principal investigator of the psychophysiology component of the project.

Address correspondence to: Nestor L. Lopez-Duran, Ph.D., University of Michigan, 530 Church St., Ann Arbor, MI 48104. E-mail: NestorL@ umich.edu events (Kovacs \& Lopez-Duran, 2010). Therefore, the objective of the current study was to examine whether frontal alpha EEG asymmetry impacts the effects of stressful life events on depressive symptoms in children at familial risk for depression.

Exposure to stressful life events has been proposed as a risk factor in children at familial risk for depression (see Goodman \& Gotlib, 1999). Indeed, children of depressed mothers experience higher levels of episodic and chronic stress than do children of either healthy mothers or mothers with nondepressive psychiatric and medical conditions (Adrian \& Hammen, 1993). Children of depressed mothers also differ from their peers in a number of other factors that may make these children more susceptible to stress, such as abnormal endocrine functioning (Ashman, Dawson, Panagiotides, Yamada, \& Wilkinson, 2002), physiologically inflexible reactions to psychological challenges (Forbes, Fox, Cohn, Galles, \& Kovacs, 2006), and greater relative right frontal EEG activity (Dawson, Frey, Panagiotides, Osterling, \& Hessl, 1997). This has led some researchers to propose that a combination of greater exposure to life stress and greater susceptibility to stress is one mechanism in the intergenerational transmission of depression risk (Goodman \& Gotlib, 1999).

Frontal alpha EEG asymmetry may be an important moderator of the detrimental effects of stress given that frontal EEG asymmetry may reflect key affective processes associated with the stress response (see Coan \& Allen, 2004; Davidson, 1993, 2004; Davidson \& Irwin, 1999). Davidson and colleagues have suggested that frontal EEG asymmetry reflects an affective style, or a trait-like tendency towards approach- or withdrawal-related motivation 
(Davidson, 1998). Specifically, studies with children and adults indicate that greater relative left frontal activation (left-sided asymmetry) is associated with approach-related behaviors, most often in response to positively valanced stimuli (see Coan \& Allen, 2004 for a review). In contrast, greater relative right frontal activation (rightsided asymmetry) is associated with withdrawal-related behaviors in response to most, but not all, negatively valenced stimuli (see Davidson, Jackson, \& Kalin, 2000). In line with this perspective, individuals with depression show decreased relative left frontal EEG activity during both depressive and euthymic states (see Thibodeau, Jorgensen, \& Kim, 2006 for meta-analytic review) and direct manipulation of the left prefrontal cortex, via transcranial magnetic stimulation, significantly decreases depressive symptoms and increases the rate of remission in patients with depressive disorders (O'Reardon et al., 2007). Furthermore, neuroimaging research of tonic cerebral blood flow and metabolism indicate reduced left prefrontal activity in patients with major depressive disorder (e.g., Drevets, 2000; Ketter, George, Kimbrell, Benson, \& Post, 1996).

Profiles of frontal EEG asymmetry have also been linked to variability in an individual's response to his/her environment. For example, greater relative right frontal EEG activation is associated with exacerbated behavioral and emotional responses to a variety of stressful stimuli and situations, such as maternal separation (Davidson \& Fox, 1989) and fear-inducing films (Tomarken et al., 1990). In contrast, greater relative left frontal EEG activation is associated with elevated positive affect in response to a positively valanced stimuli (Wheeler, Davidson, \& Tomarken, 1993), as well as an attenuated startle response to negatively valenced stimuli (Jackson et al., 2003). Not surprisingly, greater relative right frontal EEG activation has also been directly linked to the modulation of the neuroendocrine stress response in animals and humans. For example, rhesus monkeys with greater relative right frontal activation have significantly higher basal cortisol levels than their peers (Kalin, Larson, Shelton, \& Davidson, 1998). Likewise, higher cortisol reactivity to a stressor has been associated with greater relative right frontal activation in human infants (Buss et al., 2003).

Children at familial risk for depression display profiles of frontal EEG asymmetry that have been associated with an exacerbated response to stress (greater relative right activation) (Dawson et al., 1997; Field, Pickens, Fox, Nawrocki, \& Gonzalez, 1995; Jones, Field, \& Almeida, 2009; Jones, Field, \& Davalos, 2000; Jones, Field, Fox, Lundy, \& Davalos, 1997). Yet, it is unknown whether profiles of frontal EEG asymmetry influence the effect of stressful life events on depression among these children. Kovacs and Lopez-Duran (2010) recently argued that children at risk for depression have difficulties regulating sadness in response to stressful situations, and that this deficit is associated with motivational and affective processes. Specifically, the failure to regulate dysphoria among high-risk children is hypothesized to be partly due to reduced levels of positive affectivity and approach motivation (see Olino et al., 2011), which leads to a failure to deploy regulatory strategies that are dependent upon the child's ability to experience positive affect (e.g., distraction). Under this hypothesis, in the presence of stressful events, children with greater relative left frontal activation may be better equipped to effectively regulate their distress than children with greater relative right frontal activation.

Therefore, the present study examined whether frontal alpha EEG asymmetry during a series of emotion-eliciting tasks moderated the effects of life stress on early depressive symptoms in a group of children at familial risk for depression and low-risk peers. These subjects participated in a large Program Project of risk factors for childhood-onset depression but who have not yet developed depression. Thus, we examined internalizing problems as an index of depression symptoms. We hypothesized that children at high risk for depression would experience significantly more stressful life events and would show greater relative right frontal EEG activation when compared to low-risk peers. We further hypothesized that frontal EEG asymmetry during an emotion-eliciting task would moderate the association between stressful life events and internalizing symptoms. Specifically, stressful life events would be associated with greater internalizing problems among children displaying greater relative right frontal activation compared to children showing greater relative left frontal activation. Finally, we expected that the moderating effects of frontal EEG asymmetry on stressful life events would be stronger in the high-risk group compared to low-risk peers due to the greater exposure to stressful life events among the high-risk group. Exploratory analyses were conducted using other frequency bands (theta and beta) and nonfrontal electrodes to assess the specificity of our results to indices of frontal alpha EEG asymmetry.

\section{Methods}

\section{Participants}

Participants included 135 children between the ages of 6 and 13, participating in a Program Project on risk factors for childhoodonset depression (COD). The sample included 90 children who were at high risk for depression by virtue of having one parent with a documented history of COD. The remaining low-risk peers had parents who were free of any lifetime major psychiatric diagnosis. The COD group included 54 families. Twenty-one of these families had more than one child in the study. The low-risk group included 40 families, with four families having more than one child in the study. The unequal group size of the current study reflects a higher number of offspring born to the COD parents as compared to the nonaffected parents. A summary of all demographic variables for high-risk children and their low-risk peers is presented in Table 1. The two groups did not differ in sex, age, race, handedness, or parental level of education. Only 5 children in the high-risk group and 1 in the low-risk group were taking psychotropic medication at the time of assessment.

More detailed information about the recruitment procedure for the larger longitudinal study has been published elsewhere (see Forbes, Shaw, et al., 2006). Briefly, the COD parents were recruited by: (a) recontacting individuals who had participated in past research studies as mood-disordered children, (b) advertising in outpatient psychiatric clinics and related medical settings, and (c) advertising in the community. Low-risk participants were recruited by recontacting individuals who had participated in past research studies as psychologically well children, using a geographically suitable Cole directory, and by advertising in a women and infants center. All parents were evaluated via the Interview Schedule for Children and Adolescents (ISCA; for those recruited during childhood), or the Structured Clinical Interview for DSM-IV (SCID; for those recruited during adulthood). Although the Program Project included a small sample of parents with diagnosis of bipolar disorder, our analysis included only children of parents with a history of unipolar depression (MDD or dysthymia). All of these parents had their first episode onset prior to the age of 15 . 
Table 1. Characteristics of Child Participants

\begin{tabular}{|c|c|c|c|c|}
\hline & $\begin{array}{l}\text { Low-risk } \\
(N=45)\end{array}$ & $\begin{array}{l}\text { High-risk } \\
(N=90)\end{array}$ & Chi-square & $p$ \\
\hline Age-mean $(S D)$ & $7.93(2.06)$ & $7.36(1.53)$ & $F=2.62$ & .11 \\
\hline Sex (\% male) & 55.56 & 51.11 & 0.23 & 62 \\
\hline Race (\% Caucasian) & 54.44 & 55.56 & 0.01 & .90 \\
\hline Child handedness (\% strong left) & 2.22 & 4.44 & 0.41 & .47 \\
\hline Parental education level ( $\%$ high school diploma or above) & 91.11 & 93.33 & 0.19 & .65 \\
\hline Currently on psychotropic medication (\%) & 2.22 & 5.56 & 0.38 & .53 \\
\hline Stressful life events (mean) & 4.31 & 7.17 & $T=5.58$ & $<.001$ \\
\hline
\end{tabular}

\section{Measures}

Symptoms. Internalizing behavior problems in the children were measured with the Child Behavior Checklist (CBCL) for 4- to18year-olds, a parent-completed questionnaire with well established psychometric proprieties (Achenbach, 1991). This questionnaire was completed by one parent at the time of the laboratory visit (see Procedures below). For high-risk children, the questionnaire was most often completed by the parent with a history of depression. A parent rated the child on items describing the child's behavior within the previous 2 months. The CBCL yields two broad dimensions of behavior; namely, internalizing problems (anxiety, depression, and withdrawal) and externalizing problems (aggressive and destructive behaviors). Although our main interest was to examine EEG asymmetry and life stress as they relate to internalizing symptoms, we included a secondary analysis with externalizing symptoms to examine whether our proposed effects were specific to internalizing symptoms.

Stressful life events. Stressful life events were obtained during a fully structured clinical interview with the parent via the Intake General Information Sheet (IGIS). The IGIS is an interview-based questionnaire covering family demographics and health history, development, psychosocial history, and a range of stressful life events. For each item, the clinician asked the parent whether the child experienced the event within the past year or more than a year prior to the interview. The present article considers exposure to 24 stressful events (e.g., psychiatric or alcohol-related hospitalization of the parent, parental separation or divorce, loss of home, victim of sexual abuse, etc.). For our analysis, a lifetime (any time prior to the assessment) sum of all events was created with a possible range of 0 to 24. This stressful life events interview has been found to have good criterion validity, as it differentiates between depressed pediatric outpatients and school-based controls (Mayer et al., 2009).

Handedness. Participant's handedness was determined with a child version of the 11-item Edinburgh Handedness Inventory (Oldfield, 1971). We used a score of -50 to identify strong lefthanded participants. Only 5 strong left-handed participants were identified (3.7\% of the sample), and these children were included in the analyses.

\section{Procedures}

Children completed a laboratory session consisting of a series of affect-inducing tasks (see Forbes, Shaw, et al., 2006). The children first completed resting EEG recordings involving six 30-s segments. During this phase, the children alternated between looking at a picture of an interesting toy and keeping their eyes closed. The picture of the toy was used in order to keep the child's attention focused on a fixed point. Child participants were then presented with four video clips, varying in length from 57 to $189 \mathrm{~s}$, selected from popular child-appropriate films (e.g., happy: "Wizard of Oz," sad: "Lion King") in counterbalanced order. These video clips were chosen after being rated for emotional content by 25 same-age peers aged 3 to 7 (mean 4.6 years) on a scale of 1 to 3 to indicate how much the clip made them feel the target emotion $(1=$ not at all, 3 = a lot). The means for the clips used were 2.77 and 2.0 for happy and sad clips, respectively. Each clip was preceded by a brief description of the clip using a prerecorded voice. In this study, we focused analyses on profiles of frontal EEG asymmetry during the sad and happy clip.

EEG recording and quantification. For a detailed review of EEG recording and reduction procedures, see Vuga, Fox, Cohn, Kovacs, and George (2008). Resting EEG data were obtained during three 30 -s periods with eyes open and three 30 -s periods with eyes closed. These periods occurred in alternating order starting with eyes open. No difference in asymmetry scores between the eyes closed and eyes open condition were observed. EEG data were then obtained during the four film clips. EEG was recorded using 14 (12 homologous) electrodes placed in a stretch-lycra electrode cap (Electrocap, Eaton, $\mathrm{OH}$ ) positioned according to the International 10-20 System (American Electroencephalographic Society, 1994). Specifically, EEG was recorded from midfrontal (F3/F4), lateral frontal (F7/F8), central (C3/C4), posterior temporal (T7/T8), parietal (P3/P4), and occipital (O1/O2) scalp regions. Electrode impedances were required to be below $5 \mathrm{k} \Omega$ with pairs of homologous sites within $0.5 \mathrm{k} \Omega$ of each other. The bioamplifier was set for bandpass filtering with half-power cut-off frequencies of 0.01 and $100 \mathrm{~Hz}$. Artifacts were removed using an automated routine that excluded periods above a $180 \mu \mathrm{V}$ threshold. This procedure was verified against a manual review in 44 participants. Intraclass correlations of EEG alpha power between the two methods ranged from .94 to 1 . Artifact-free EEG data were then rereferenced to a common average reference. Participants had sufficient artifact-free data (above $70 \%$ for all conditions), and therefore no subjects were excluded due to excessive artifacts. The segments were not weighed based on the level of artifact-free data. Fourier analyses were applied to all epochs (30-s for baseline and variable for film conditions) using 1-s artifact-free Hanning-windowed data with $50 \%$ overlap in each epoch. Power spectral density $\left(\mu \mathrm{V}^{2} / \mathrm{Hz}\right)$ were computed for the alpha, theta, and beta bands. Due to differences in power distribution in young children, different alpha bands were used for different age groups to account for age-specific spectral alpha activities based on their age-dependent peak frequencies (see 


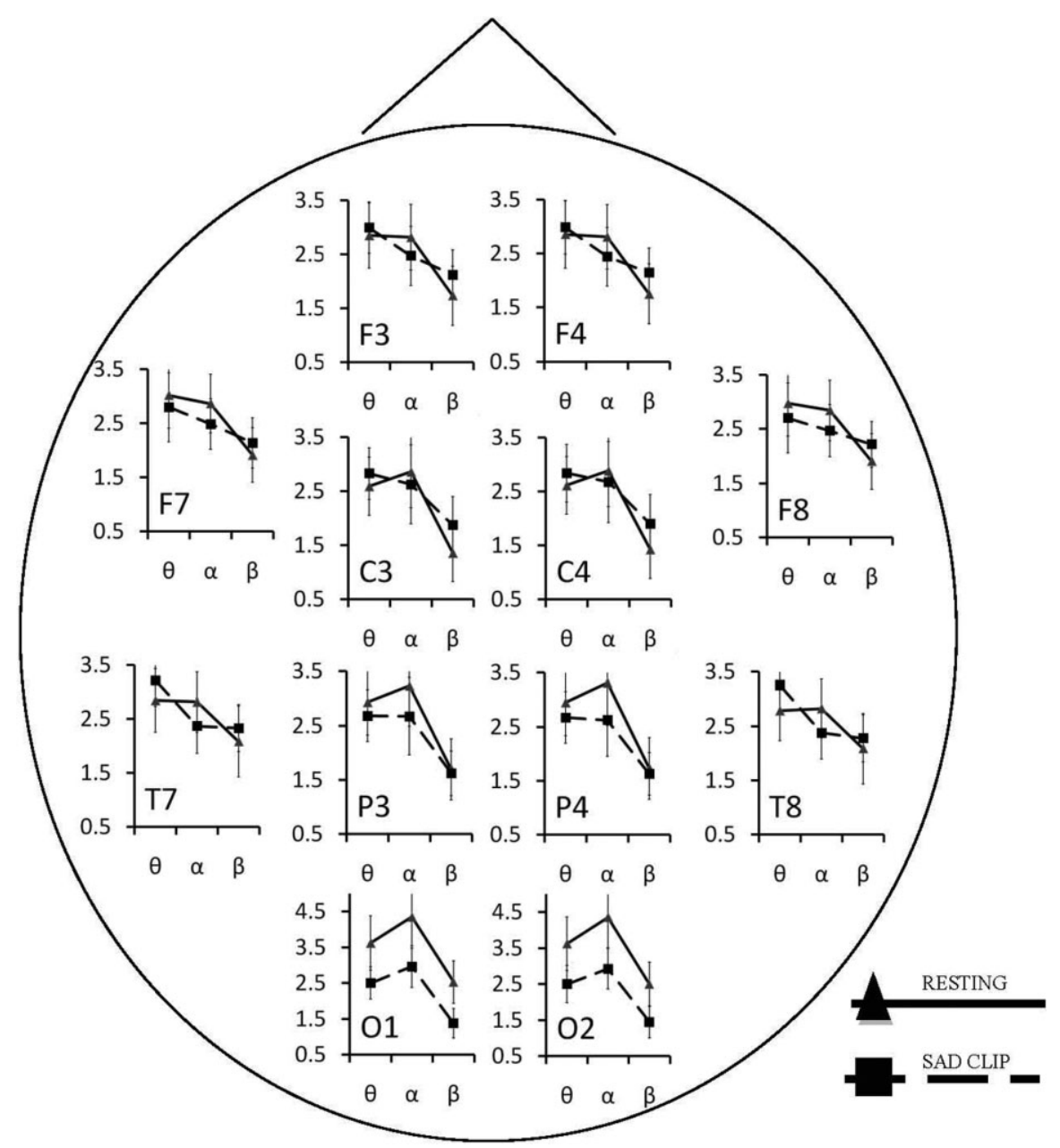

Figure 1. Natural log power in the theta, alpha, and beta ranges for the resting and sad film in all electrode locations for all children. Y axis refers to natural $\log$ power. $\mathrm{X}$ axis includes theta, alpha, and beta bands. Error bands extend 1 standard deviation from each mean.

Marshall, Bar-Haim, \& Fox, 2002; Vuga et al., 2008). Specifically, in accordance with power distribution in preschool children (Coan \& Allen, 2004; Marshall et al., 2002), alpha corresponded to 6.5 to $10.5 \mathrm{~Hz}$ in 3 - to 5 -year-olds. We also shifted the alpha band by $1 \mathrm{~Hz}$ from preschool children to school-age children based on the difference in their age-dependent peak frequencies $(8 \mathrm{~Hz}$ in preschool children; Niedermeyer, 1999). Therefore, alpha corresponded to 7.5 to $11.5 \mathrm{~Hz}$ in 6- to 9-year-olds. These alpha band definitions have been used in previous research by Forbes, Shaw, et al. (2006) and Vuga et al. (2008). We also used similar shifts for theta (3.0 to $5.5 \mathrm{~Hz}$ in 5 -year-olds, 4 to $6.5 \mathrm{~Hz}$ in 6- to 9-year-olds) and beta (11-16.5 in 5-year-olds, $12-17.5$ in 6- to 9-year olds) in computing power and asymmetry at these bands. Figure 1 plots the natural log-transformed power scores for the alpha, theta, and beta frequency bands at each electrode both at rest and during the sad film clip. Natural log-transformed alpha power values in the present study are consistent with existing research of alpha power in children (e.g., Jones et al., 2000).

Asymmetry scores were derived from the difference of natural log-transformed power scores (Gasser, Bächer, \& Möcks, 1982) for the right minus the left sites (e.g., $\ln F 8-\operatorname{lnF} 7$ ) (Davidson et al., 2000) for all homologous electrodes. Hypotheses focused on asymmetry indices in the alpha band given that higher alpha power reflects lower brain activity (Davidson, Ekman, Saron, Senulis, \&
Friesen, 1990). Accordingly, positive alpha asymmetry scores indicate greater relative left than right activity. Also in line with existing research, hypotheses focused on midfrontal (F3/F4) and lateral frontal $(\mathrm{F} 7 / \mathrm{F} 8)$ regions because these regions have been consistently associated with stress, affect, and depression (Coan \& Allen, 2004; Lewis, Weekes, \& Wang, 2007; Tops et al., 2005). Exploratory analyses were conducted for nonfrontal asymmetry indices in the alpha band, as well as both frontal and nonfrontal asymmetry indices in the theta and beta bands, to determine whether any observed results were specific to the frontal alpha asymmetry indices.

Data analysis. Mixed random effects models (SAS PROC MIXED with ML estimation) were used to examine the effects of risk status, asymmetry, stressful life events, and the 2- and 3-way interactions between these terms as predictors of internalizing problems. Separate models were conducted for each condition (baseline, happy film clips, sad film clips) and each frontal asymmetry index (F7/F8, F3/F4). Since the COD and nonaffected groups included a number of families with siblings (21 and 4 , respectively), we entered Family as a random effect into all models. All models that used frontal asymmetry during the happy or sad clip as predictors also included the corresponding resting frontal asymmetry index as a control. Interaction effects were examined by 
Table 2. Unadjusted Pearson's Correlations for All Variables

\begin{tabular}{|c|c|c|c|c|c|c|c|c|c|c|}
\hline & 1 & 2 & 3 & 4 & 5 & 6 & 7 & 8 & 9 & 10 \\
\hline \multicolumn{11}{|l|}{ 1. CBCL internalizing } \\
\hline 2. Life events & $.47 * *$ & & & & & & & & & \\
\hline 3. Age & .16 & $.36^{* *}$ & & & & & & & & \\
\hline 4. Handedness & .12 & $.19 *$ & $.21 *$ & & & & & & & \\
\hline 5. Parental education & $-.21 *$ & $-.22 *$ & .08 & .15 & & & & & & \\
\hline 6. F3/F4 baseline & .09 & .00 & .06 & $.22 *$ & $.29 * *$ & & & & & \\
\hline 7. F3/F4 happy clip & .12 & -.01 & .16 & .14 & $.26 * *$ & $.64 * *$ & & & & \\
\hline 8. F3/F4 sad clip & .03 & .01 & .19 & .11 & $.32 * *$ & $.70 * *$ & $.78 * *$ & & & \\
\hline 9. F7/F8 baseline & .09 & -.08 & -.12 & .07 & .09 & $.51 * *$ & $.26 * *$ & $.22 *$ & & \\
\hline 10. F7/F8 happy clip & -.07 & $-.26^{* *}$ & -.18 & -.05 & .17 & $.32 * *$ & $.37 * *$ & $.34 * *$ & $.62 * *$ & \\
\hline 11. F7/F8 sad clip & -.04 & $-.25^{*}$ & -.11 & -.07 & $.19 *$ & $.34 * *$ & $.37 * *$ & $.46^{* *}$ & $.56 * *$ & $.81 * *$ \\
\hline
\end{tabular}

F3/F4 = Midfrontal alpha EEG asymmetry; F7/F8 = Lateral frontal alpha EEG asymmetry; CBCL = Child Behavior Check List.

$* p<.05 . * * p<.01$.

comparing models with decreasing levels of parsimony using a hierarchical framework; namely, models containing Model 1: main effects; Model 2: main effects and 2-way interactions; and Model 3: main effects, 2-way, and 3-way interactions. We examined changes in model fit using the Akaike Information Criterion (AIC) and the Bayesian Information Criterion (BIC) after the addition of the 2- and 3-way interactions before we conducted post hoc analyses. A decrease in the AIC and BIC in nested models of increasing complexity (more parameters) is interpreted as indicative of an improvement in model fit (Bozdogan, 1987; Pan, 2001). Therefore, we examined the nature of the interactions only when a model showed improvement in fit, based on at least one of these fit indices. Interpretations of significant higher order interactions were conducted in models that included all lower order interactions. Significant asymmetry effects were followed by an individual hemisphere analysis based on a revised residualized power approach (Allen, Coan, \& Nazarian, 2004). To test the specificity of EEG main effects and interactions to both the frontal asymmetry indices and the alpha frequency band, we performed exploratory analyses by replacing the asymmetry variables in the "best fit" model identified above for the same task with (a) asymmetry indices for alpha power for nonfrontal electrodes, and (b) asymmetry indices for theta and beta frequency bands.

\section{Results}

\section{Descriptive Analyses}

Stressful life events. The high-risk group experienced significantly more stressful life events than the low-risk group (high-risk mean $=7.17, S D=3.64$ vs. control mean $=4.31, S D=2.29$ ), $t(126)=5.58, p<.01$. Among the high-risk group, $75 \%$ of the offspring $(N=68)$ had been exposed to 5 or more stressful life events. In contrast, among the low-risk peers, only $34 \%$ of the sample $(N=15)$ experienced 5 or more life events. Stressful life events were associated with parental levels of education $(r=-.22$, $p=.01)$ and age $(r=.36, p<.01)$, but were not associated with sex, $t(130)=.38, p=.70$.

Frontal alpha EEG asymmetry. Table 2 presents the unadjusted correlations of frontal alpha EEG asymmetry, stressful life events, child's age, child's handedness, and parental levels of education. Parental education was associated with a tendency towards greater relative left midfrontal activation during all conditions (baseline, happy, sad), and greater relative left lateral frontal activation during the sad condition. The child's age, sex, race, handedness, and whether the child was taking psychotropic medications at the time of the assessment were not associated with frontal EEG asymmetry in any of the testing conditions.

Table 3 presents the means, standard deviations, and effect sizes of mid- and lateral frontal EEG asymmetry in our target sites for the high-risk and low-risk participants. A series of mixed effects models were conducted to examine the differences in frontal EEG asymmetry between the high-risk and low-risk participants. Highrisk participants displayed greater relative right lateral frontal activation (F7/F8) compared to the low-risk children during the happy (Cohen's $d=-.47$ ) and sad ( $d=-.50)$ film clips. No group difference in lateral frontal asymmetry was noted during the resting condition, or for the midfrontal asymmetry index (F3/F4) at rest or during any of the film clips.

\section{Associations with Internalizing Symptoms: Stressful Events and Risk Status}

Results of a hierarchical mixed effects models indicated that stressful life events predicted higher levels of internalizing symptoms, $b=1.12, t(35)=4.17, p=.002$. Risk status also predicted internalizing symptoms, $b=6.89, t(92)=3.43, p=.009$, with high-risk children having significantly higher internalizing symptoms than their peers (high-risk $M=55.74, S D=11.12$; low-risk $M=45.56$,

Table 3. Alpha EEG Asymmetry for High-Risk and Low-Risk Children in All Experimental Conditions

\begin{tabular}{lcrrrr}
\hline \hline & $\begin{array}{c}\text { High-risk } \\
\text { mean* }(S D)\end{array}$ & $\begin{array}{c}\text { Low-risk } \\
\text { mean* }(S D)\end{array}$ & \multicolumn{1}{c}{$F$} & $p$ & $d$ \\
\hline Resting & & & & & \\
F3/F4 & $-.014(.149)$ & $.012(.105)$ & 1.02 & .30 & -0.21 \\
F7/F8 & $-.028(.136)$ & $-.008(.121)$ & 2.14 & .14 & -0.16 \\
Happy clip & & & & & \\
F3/F4 & $-.034(.160)$ & $-.027(.122)$ & .07 & .79 & 0.05 \\
F7/F8 & $-.034(.146)$ & $.029(.118)$ & 4.82 & .03 & -0.48 \\
Sad clip & & & & & \\
F3/F4 & $-.033(.182)$ & $-.009(.135)$ & .62 & .43 & -0.15 \\
F7/F8 & $-.036(.147)$ & $.035(.134)$ & -5.25 & .02 & -0.50 \\
\hline \hline
\end{tabular}

*Negative values indicate greater relative right frontal EEG activation. F3/F4 = Midfrontal alpha EEG asymmetry; F7/F8 = Lateral frontal alpha EEG asymmetry. 
Table 4. Hierarchical Modeling of Internalizing Problems as a Function of Midfrontal (F3/F4) Asymmetry, Risk Status, and Life Events

\begin{tabular}{|c|c|c|c|c|c|c|c|c|c|c|c|c|}
\hline & \multicolumn{4}{|c|}{ Resting } & \multicolumn{4}{|c|}{ Happy clip ${ }^{\dagger}$} & \multicolumn{4}{|c|}{ Sad clip ${ }^{\dagger}$} \\
\hline & $b$ & $p$ & $\mathrm{AIC}$ & $\mathrm{BIC}$ & $b$ & $p$ & $\mathrm{AIC}$ & $\mathrm{BIC}$ & $b$ & $p$ & $\mathrm{AIC}$ & $\mathrm{BIC}$ \\
\hline \multicolumn{13}{|l|}{ Models } \\
\hline Main effects & & & 931.6 & 946.8 & & & 715.9 & 732.1 & & & 707.7 & 724 \\
\hline Asymmetry (F3/F4) & 6.11 & .32 & . & & 9.87 & .191 & & & 2.80 & .981 & & \\
\hline Risk status (high-risk) & 7.57 & .000 & & & 6.26 & .011 & & & 5.82 & .018 & & \\
\hline Life events & 1.05 & .000 & & & 1.01 & .003 & & & 1.10 & .002 & & \\
\hline 2-Way interactions & & & 934 & 954.4 & & & $715.8 *$ & 736.7 & & & 709.0 & 729.9 \\
\hline Asymmetry (F3/F4) & -3.11 & .86 & & & 14.88 & .358 & & & -18.20 & .253 & & \\
\hline Risk status (high-risk) & 7.21 & .000 & & & 6.81 & .006 & & & 5.94 & .017 & & \\
\hline Life events & 1.10 & .000 & & & 0.90 & .009 & & & 1.17 & .002 & & \\
\hline Asymmetry $\times$ life events & 2.87 & .21 & & & -3.25 & .081 & & & 1.55 & .514 & & \\
\hline Asymmetry $\times$ risk status & -11.59 & .51 & & & 23.67 & .151 & & & 14.04 & .362 & & \\
\hline 3-Way interactions & & & $933.8^{*}$ & 956.7 & & & $715.4 *$ & 738.5 & & & 709.5 & 732.6 \\
\hline Asymmetry (F3/F4) & -40.15 & .19 & & & -25.49 & .409 & & & -52.53 & .109 & & \\
\hline Risk status (high-risk) & 7.04 & .000 & & & 7.40 & .003 & & & 6.46 & .011 & & \\
\hline Life events & 1.07 & .000 & & & 0.89 & .009 & & & 1.06 & .006 & & \\
\hline Asymmetry $\times$ life events & 11.99 & .07 & & & 5.92 & .341 & & & 10.37 & .170 & & \\
\hline Asymmetry $\times$ risk status & 34.45 & .33 & & & 71.24 & .051 & & & 60.51 & .135 & & \\
\hline Asymmetry $\times$ risk $\times$ life events & -10.39 & .14 & & & -9.97 & .131 & & & -10.11 & .213 & & \\
\hline
\end{tabular}

*Significant improvement in model fit.

All models included baseline alpha asymmetry as control.

$S D=8.37 ; d=1.04)$. There was no interaction between stressful life events and risk status in predicting internalizing symptoms (main effects model AIC 958.6 vs. main effects plus interaction model AIC 960.5).

\section{Associations with Internalizing Symptoms: Frontal Alpha EEG Asymmetry}

All hierarchical mixed effects models presented below include both left- and right-handed children. However, we also replicated these models using strongly right-handed children only (Edinburgh Handedness Inventory cut-off $=50$ ) and observed identical overall results. Consistent with the results reported above, risk status and stressful life events were significant predictors of internalizing symptoms in all models. Although these variables were included in all models, below we only describe results for the main affects of asymmetry and related interactions.

Midfrontal (F3/F4) EEG asymmetry as a predictor of internalizing symptoms. Table 4 presents the results of all hierarchical models exploring the association between midfrontal asymmetry, risk status, and stressful life events as predictors of internalizing symptoms. Midfrontal asymmetry was not associated with internalizing symptoms regardless of clip condition. Midfrontal asymmetry did not interact with stressful life events and/or risk status in any of the clip conditions.

Lateral frontal (F7/F8) EEG asymmetry as a predictor of internalizing symptoms. Table 5 presents the results of all hierarchical models exploring the association between lateral frontal alpha asymmetry, risk status, and stressful life events as predictors of internalizing symptoms.

Resting lateral frontal EEG asymmetry. The main effects model suggested that lateral frontal asymmetry at rest was not associated with internalizing symptoms. A model with the 2-way interactions showed improvement in model fit (main effects model AIC 931.7 vs. 2-way interaction model AIC 930.1). However, the interaction between lateral frontal asymmetry at rest and stressful life events was not significant, $b=1.02, t(30)=-.41, p=.68$, and the interaction between lateral frontal asymmetry at rest and risk status only approached significance, $b=-29, \quad t(30)=-1.79$, $p=.08$. The 3 -way interaction model also resulted in an improvement of the model fit (AIC=929.2). However, the lateral frontal asymmetry $\times$ stressful life events $\times$ risk status only approached significance, $b=-10, t(18)=-1.81, p=.09$.

Lateral frontal EEG asymmetry during the happy film. The main effects model showed no association between lateral frontal asymmetry during the happy film and internalizing symptoms while controlling for lateral frontal asymmetry at rest. A model with the 2-way interactions showed no improvement in model fit (main effects model AIC 717.8 vs. 2-way interaction model AIC 717.9), indicating no 2-way interactions. The 3-way interaction model resulted in an improvement of the model fit (AIC $=716.7)$. However, the lateral frontal asymmetry during the happy film $\times$ stressful life events $\times$ risk status interaction only approached significance, $b=-10.03, F(1,18)=3.29, p=.08$.

Lateral frontal EEG asymmetry during the sad film. The main effects model suggested that, while controlling for lateral frontal asymmetry at rest, lateral frontal asymmetry during the sad film was not associated with internalizing symptoms. A model with the 2-way interactions showed improvement in model fit (main effects model AIC 708 vs. 2-way interaction model AIC 702.7). The interaction between lateral frontal asymmetry during the sad film and stressful life events was significant, $b=-5.71, F(1,18)=9.50$, $p=.006$, indicating that lateral frontal asymmetry scores during the sad film moderated the effect of stressful life events on internalizing symptoms. Specifically, the effects of stressful life events on internalizing symptoms decreased as relative left lateral frontal activation scores increased (i.e., moving towards greater left than right lateral frontal activation). However, a significant 3-way interaction model (AIC $=699.5)$ suggested that this effect was further moderated by risk status, $b=-11.49, F(1,17)=5.43, p=.03$. Spe- 
Table 5. Hierarchical Modeling of Internalizing Problems as a Function of Lateral Frontal (F7/F8) Asymmetry, Risk Status, and Life Events

\begin{tabular}{|c|c|c|c|c|c|c|c|c|c|c|c|c|}
\hline & \multicolumn{4}{|c|}{ Resting } & \multicolumn{4}{|c|}{ Happy clip ${ }^{\dagger}$} & \multicolumn{4}{|c|}{ Sad clip ${ }^{\dagger}$} \\
\hline & $b$ & $p$ & AIC & BIC & $b$ & $p$ & AIC & BIC & $b$ & $p$ & AIC & BIC \\
\hline \multicolumn{13}{|l|}{ Models } \\
\hline Main effects & & & 931.7 & 947 & & & 717.8 & 734 & & & 708 & 724.3 \\
\hline Asymmetry & 5.78 & .358 & & & -1.36 & .852 & & & 2.39 & .736 & & \\
\hline Risk status (high-risk) & 7.58 & .000 & & & 6.17 & .01 & & & 5.75 & .022 & & \\
\hline Life events & 1.06 & .000 & & & 1.03 & .004 & & & 1.13 & .002 & & \\
\hline 2-Way interactions & & & $930.1^{*}$ & 950.4 & & & 717.9 & 738.7 & & & $702.7 *$ & $723.6^{*}$ \\
\hline Asymmetry & 33.94 & .035 & & & 23.67 & .140 & & & 32.96 & .039 & & \\
\hline Risk status (high-risk) & 7.68 & .000 & & & 6.74 & .007 & & & 5.99 & .014 & & \\
\hline Life events & 1.02 & .001 & & & 0.87 & .013 & & & 0.86 & .011 & & \\
\hline Asymmetry $\times$ life events & -0.88 & .681 & & & -3.07 & .104 & & & -5.71 & .006 & & \\
\hline Asymmetry $\times$ risk status & -29.36 & .08 & & & -9.10 & .577 & & & 8.13 & .606 & & \\
\hline 3-Way interactions & & & $929.2 *$ & 952.1 & & & 716.7 & 739.9 & & & $699.5^{*}$ & $722.7 *$ \\
\hline Asymmetry & -0.43 & .98 & & & -12.81 & .617 & & & -4.39 & .841 & & \\
\hline Risk status (high-risk) & 8.02 & .000 & & & 6.83 & .006 & & & 6.61 & .006 & & \\
\hline Life events & 0.89 & .006 & & & 0.67 & .054 & & & 0.54 & .114 & & \\
\hline Asymmetry $\times$ life events & 6.68 & .183 & & & 5.54 & .288 & & & 3.19 & .459 & & \\
\hline Asymmetry $\times$ risk status & 21.33 & .531 & & & 37.49 & .229 & & & 64.82 & .036 & & \\
\hline Asymmetry $\times$ risk $\times$ life events & -9.40 & .096 & & & -10.03 & .086 & & & -11.49 & .032 & & \\
\hline
\end{tabular}

*Significant improvement in model fit.

†All models included baseline alpha asymmetry as control.

cifically, the previously observed interaction between lateral frontal asymmetry scores and stressful life events was significant for the high-risk group, $b=-8.29, t(17)=-3.93, p=.001$, but not for the low-risk participants, $b=3.18, t(17)=0.78, p=.46$.

Due to the significant 3-way interaction, we conducted a standard post hoc analysis of the lateral frontal asymmetry moderation effect by dichotomizing the moderator (asymmetry) scores (see Baron \& Kenny, 1986). To this end, we divided the lateral frontal asymmetry scores into those with greater left than right lateral frontal activation and those with greater right than left lateral frontal activation. We then repeated the original models separately by the two levels of the moderator and conducted post hoc slope contrasts predicting internalizing symptoms from life stressors for high-risk children displaying either greater left than right activation or greater right than left activation. Increasing numbers of stressful life events was associated with increasing internalizing symptoms for high-risk children with greater right than left lateral frontal activation (slope $=1.39), t(16)=3.51, p=.003$, but not for highrisk children with greater left than right lateral frontal activation (slope $=-0.23$ ), $t(16)=-.32, p=.75$. Figure 2 presents the association between stressful life events and internalizing symptoms for high-risk children displaying both levels of the dichotomized asymmetry scores.

Analyses of individual hemisphere for lateral frontal alpha power. In line with existing research (Coan \& Allen, 2004; Thibodeau et al., 2006), our primary predictions involved relative alpha activity in the left as compared to the right hemisphere, and, thus, we computed asymmetry indices for all homologous sites. The asymmetry index controls for individual differences in skull thickness and volume conduction, which could produce differences in alpha power. A question of interest, however, concerns the contributions of each hemisphere to a significant asymmetry score. Accordingly, we conducted a series of individual hemisphere analyses for the lateral frontal electrodes (F7 and F8) to explore whether the lateral frontal asymmetry effect was due to the direct contribution of a specific hemisphere (e.g., high activation of the left lateral region as opposed to low activation of the right lateral region). To this end, we regressed internalizing symptoms on whole head alpha power (natural log-transformed alpha power at all recording sites) and saved the unstandardized residuals (see Allen et al., 2004). We then replicated the original hierarchical models separately for each hemisphere site predicting the unstandardized residuals. For example, the left hemisphere models included risk status, stressful life events, natural log-transformed alpha power of the lateral frontal region (F7), and the 2- and 3-way interactions between these factors as predictors of the unstandardized residuals. None of the main effects for either F7 alpha power or F8 alpha power, or their 2- and 3-way interactions with stressful life events and/or risk status were significant, suggesting that it is the relative relationship between the left and right lateral frontal activity (i.e., the lateral frontal asymmetry index), as opposed to alpha activity at any specific electrode, that interacts with risk status and stressful life events to predict internalizing symptoms.

Analysis of nonfrontal alpha asymmetry indices and analyses of theta and beta asymmetry indices. In line with existing research (Coan \& Allen, 2004; Thibodeau et al., 2006), our predictions focused on frontal EEG asymmetry in the alpha range. An important question of interest is whether the observed effect is unique to the alpha range and to the frontal region. To this end, we examined the stress $\times$ risk status $\times$ asymmetry interaction predicting internalizing symptoms for the nonfrontal asymmetry indices for alpha power (T7/T8, C3/C4, P3/P4, O1/O2). We also examined the stress $\times$ risk status $\times$ symmetry interaction predicting internalizing symptoms for the mid- and lateral frontal asymmetry indices for the theta and beta frequency bands, as well as for the nonfrontal asymmetry indices for theta and beta power (T7/T8, C3/C4, P3/P4, $\mathrm{O} 1 / \mathrm{O} 2)$.

Stress and risk status did not interact with the lateral frontal asymmetry scores $(\mathrm{F} 7 / \mathrm{F} 8)$ in the theta $F(1,17)=0.02, p>.10$ or beta $F(1,17)=0.31, p>.10$ bands; temporal asymmetry scores 


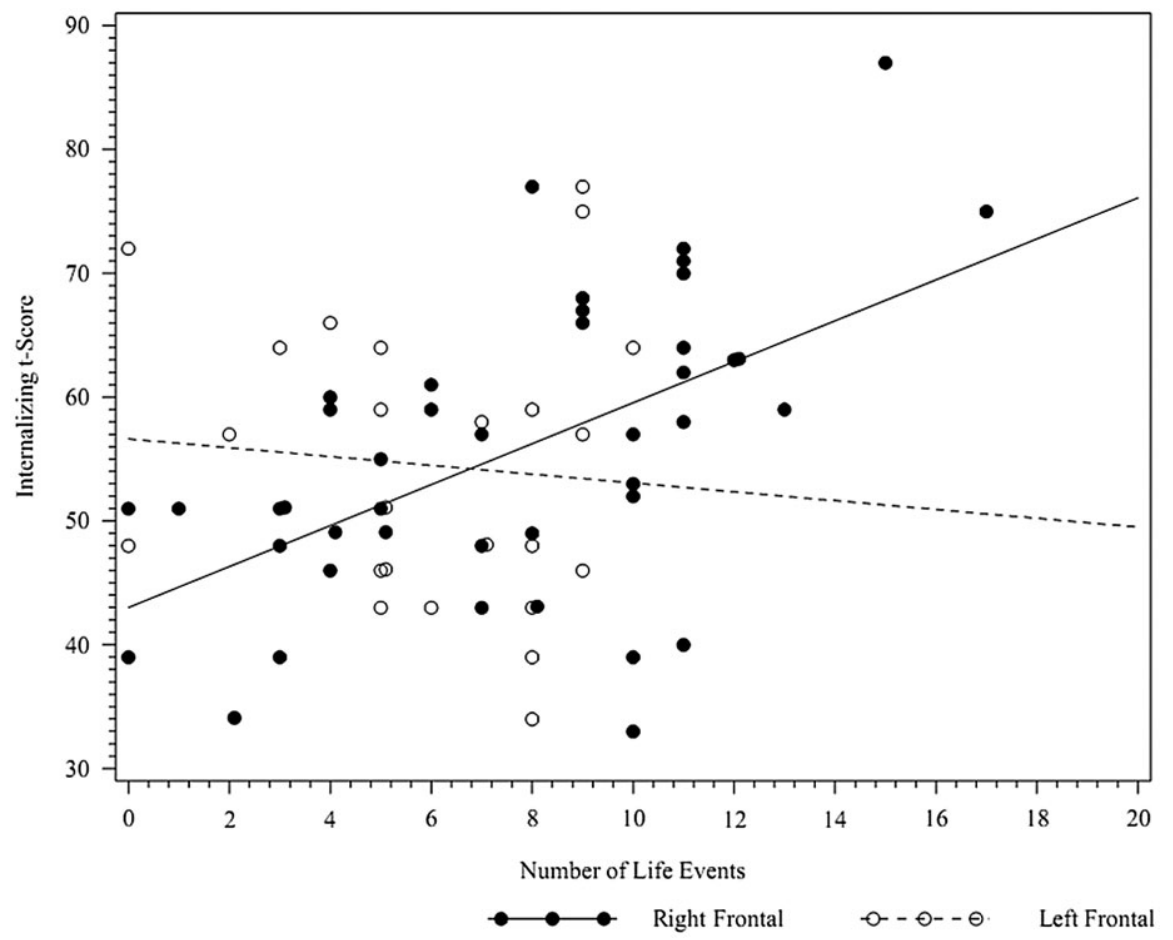

Figure 2. Internalizing scores by number of life events for high-risk children with greater left than right ("Left Frontal") versus greater right than left ("Right Frontal") lateral frontal (F7/F8) activation during a sad clip.

(T7/T8) in the theta $F(1,17)=0.19, p>.10$, alpha $F(1,18)=0.01$, $p>.10$, or beta $F(1,1)=0.00, p>.10$ bands; central asymmetry scores $(\mathrm{C} 3 / \mathrm{C} 4)$ in the theta $F(1,17)=0.01, p>.10$, alpha $F(1,17)=0.05, p>.10$, or beta $F(1,17)=0.00, p>.10$; parietal asymmetry scores $(\mathrm{P} 3 / \mathrm{P} 4)$ in the theta $F(1,17)=0.29, p>.10$, alpha $F(1,17)=1.01, p>.10$, or beta $F(1,17)=1.46, p>.10$; or occipital asymmetry scores $(\mathrm{O} 1 / \mathrm{O} 2)$ in the theta $F(1,17)=0.00$, $p>.10$, alpha $F(1,17)=0.43, p>.10$, or beta $F(1,17)=2.35$, $p>.10$ bands. Figure 3 presents the asymmetry scores for all regions and EEG bands among children categorized based on their risk status, internalizing symptoms, and stress exposure.

\section{Discussion}

In the current study, we examined the relation among frontal alpha EEG asymmetry, internalizing problems, and life stressors in children at familial risk for depression and their low-risk peers. We hypothesized that high-risk children would have higher levels of life stress, internalizing problems, greater relative right frontal EEG activity, and that frontal EEG asymmetry would moderate the effects of life stress on internalizing problems. The results partially support our hypotheses. As expected, and consistent with previous studies (Adrian \& Hammen, 1993), we found that high-risk children experienced greater numbers of stressful life events and more internalizing problems than their low-risk peers, which has been proposed as one potential mechanism for the intergenerational transmission of depression risk (Goodman \& Gotlib, 1999). We further found that high-risk children had significantly greater relative right lateral frontal EEG activation than the low-risk children, but only during the happy and sad films. Specifically, high-risk children displayed greater relative right lateral frontal EEG activation during both films while the low-risk group displayed greater relative left lateral frontal EEG activation during both films. Furthermore, our results suggest that lateral frontal asymmetry scores may moderate the effects of exposure to stressful life events in the high-risk children, such that greater relative left lateral frontal activation could mitigate the effects of stressful events on internalizing symptoms.

This is the first study to show differences in frontal alpha EEG asymmetry between elementary school-aged high-risk children and their low-risk peers while watching emotionally evocative films. This extends the findings of a large number of studies using infants of currently depressed mothers (Dawson et al., 1997; Field, Pickens, Fox, \& Nawrocki, 1995; Jones et al., 2000, 2009; Jones, Field, Davalos, \& Pickens, 1997), suggesting that this effect may be developmentally stable and represents a possible vulnerability. This effect was observed in the happy and sad films but not in the baseline condition. This film-specific effect is consistent with the capability model proposed by Coan, Allen, and McKnight (2006), suggesting that frontal asymmetry during emotionally salient events is a more robust predictor of an individual's capabilities in approach/withdrawal motivation. However, our findings are contrary to other studies with children of depressed parents who reported differences in frontal asymmetry at rest (Dawson et al., 1997; Field, Pickens, Fox, \& Nawrocki, 1995; Jones, Field, Davalos, \& Pickens, 1997; Jones et al., 2000, 2009). The discrepancy may be due to methodological and age differences between the studies. All but one of previous studies used infants of currently depressed mothers and included a resting condition that may not be equivalent to the resting condition used in our study. For example, in Jones et al. (2009), infants were shown their favorite toy to keep them sitting quietly with their eyes opened. The only noninfant study (Tomarken, Dichter, Garber, \& Simien, 2004) included only adolescents, 


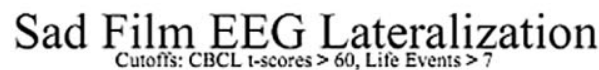

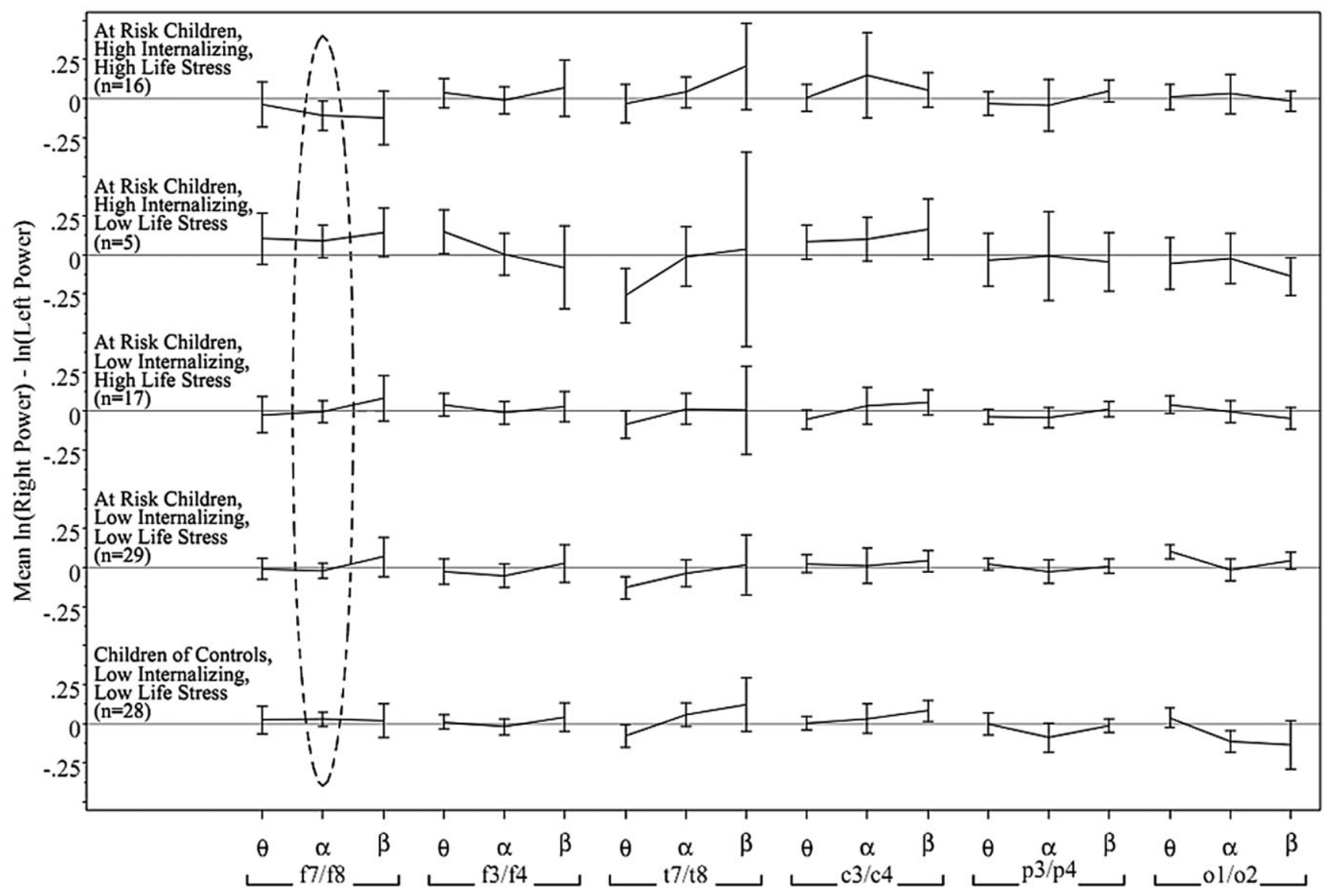

Electrode Pairs and Band

Figure 3. EEG asymmetry profiles across scalp regions and EEG band in participant subgroups while watching a sad film. Lines connect mean asymmetry in adjacent bands from the natural log power spectrum in the same electrode pair and subgroup; error bars extend \pm 2 standard errors from each mean. The horizontal reference is at zero asymmetry for each subgroup. Positive values indicate greater left than right activation. The dashed circle highlights the only significant observed risk status $\times$ asymmetry $\times$ life event interaction predicting internalizing symptoms after adjusting for baseline asymmetry. This effect can be seen in the F7/F8 alpha asymmetry where the topmost subgroup (at-risk children with high internalizing scores with high life stress) tend to display greater relative right lateral frontal activity, in contrast to the middle subgroup (at-risk children with low internalizing scores with high life stress) whose mean asymmetry is near zero. This 3-way interaction was not significant in any of the other electrode pairs or frequency bands.

which may differ from our sample on key-related variables (e.g., number and severity of depressive symptoms).

The difference in frontal EEG asymmetry between the highand low-risk participants was specific to the lateral frontal region (F7/F8). Consistent with this finding, greater relative right lateral frontal, but not midfrontal, EEG activity has been associated with higher negative emotionality in adults (Jacobs \& Snyder, 1996) and with stronger cortisol responses and fear expressions among infants during a stranger paradigm (Buss et al., 2003). However, other studies with adults have linked midfrontal, but not lateral frontal, asymmetry scores to constructs associated with affective style and emotion regulation, such as the Behavioral Activation/Inhibition System (Coan \& Allen, 2003) and recovery (startle magnitude) after exposure to negatively valenced pictures (Jackson et al., 2003). These inconsistencies may be due to a number of differences between past studies (e.g., age, sample characteristics, experimental conditions, outcomes), and highlight the need for additional examinations of the role of mid- versus lateral frontal EEG asymmetry in the development of depression among at-risk individuals.

In addition, high-risk participants displayed a stable pattern of greater relative right lateral frontal EEG activation during all conditions (see Table 3). In contrast, the low-risk participants displayed a marked shift from minimal asymmetry during the baseline to greater relative left lateral frontal activation during the happy and sad films. Therefore, there was no apparent difference in frontal asymmetry patterns between the two emotion films in either group, which is contrary to some, but not all, previous examinations of EEG responses to emotion films (e.g., Davidson \& Fox, 1982; Davidson et al., 1990; Reeves, Lang, Thorson, \& Rothschild, 1989). Given that the films were all selected from popular children's films, it is possible that among the low-risk children, all films elicited an approach-oriented state as reflected in the shift towards greater relative left lateral frontal activation. In contrast, 
the at-risk group showed no change in frontal asymmetry to the films, possibly reflecting a blunted affective response to these stimuli. This would be in line with recent studies showing that children at familial risk for depression have reduced positive affect than their low-risk peers during play activities (Olino et al., 2011).

Frontal alpha EEG asymmetry during a sad film moderated the effects of stress in the high-risk group, who were also exposed to a significantly higher number of stressful events than their low-risk peers. Specifically, as frontal asymmetry moved towards greater relative left frontal activation, the association between stressful life events and internalizing symptoms decreased. Post hoc analyses of the moderating effect suggests a protective function of greater left than right frontal EEG activation rather than a risk imposed by greater right than left frontal activation. Specifically, we found that those high-risk children who had greater right than left frontal activation displayed the same relationship between life stress and internalizing symptoms observed in their low-risk peers: greater life stressors were associated with higher internalizing symptoms. In contrast, high-risk children with greater left than right frontal activation showed no association between stressful life events and internalizing symptoms.

Moreover, while the interaction of frontal asymmetry and stressful life events was observed only during the sad film, similar patterns were noted for the resting condition and the happy film, although not at the statistically significant level. This suggests that our findings may not be specific to responses to sadness. Instead, since it is likely that both films elicited approach-related affect in the low-risk group, our results suggest that it is the general ability for positive affect and approach motivation, as reflected in greater relative left frontal activation during the films, that protects at-risk children from the effects of stressful events.

The moderating effect of frontal EEG asymmetry on life stress is in line with theories of affective styles and motivation. Davidson and colleagues have argued that individuals with greater relative right frontal activation have an affective style characterized by more dispositional negative affect and more intense responses to negative affective challenges (see Davidson, 1998). In contrast, greater relative left frontal activation is associated with positive emotional expressions in response to emotionally evocative films of different valence (Allen, Harmon-Jones, \& Cavender, 2001). Therefore, greater relative left frontal activation may also reflect a high capacity to experience pleasure (i.e., hedonic capacity), which serves a key function in emotion regulation (see Kovacs \& LopezDuran, 2010). That is, youngsters with high hedonic capacity and approach motivation may tend to deploy more effective regulatory mechanisms in response to stressful events than their peers. For example, in a separate study with the current sample, high-risk children who had greater relative right frontal activation, compared to their low-risk peers, were less likely to engage in proactive regulatory behaviors (e.g., distraction), and more likely to wait passively and focus on the aversive stimuli during a laboratory stress task (Silk, Shaw, Skuban, Oland, \& Kovacs, 2006).

There is also evidence, albeit equivocal (see Lewis et al., 2007), that greater relative right frontal activation modulates the neuroendocrine response to stressors (Buss et al., 2003; Kalin et al., 1998; Tops et al., 2005), and such endocrine response has been implicated in the development of internalizing problems and depression (for a review, see Lopez-Duran, Kovacs, \& George, 2009; LopezDuran, Vazquez, Felt, \& Olson, 2009). Therefore, it is possible that among high-risk children, greater relative left frontal activation facilitates a blunted endocrine response to high levels of stress, which may prevent the development of internalizing symptoms by reducing the subjective experience of stress (see Smagin, Heinrichs, \& Dunn, 2001), limiting the consolidation of stress-related memories (see Buchanan \& Lovallo, 2001), or limiting exposure to high and chronic amounts of corticoids (see Lopez-Duran, Vazquez, et al., 2009).

Notably, we did not find an interaction between stress and risk status predicting internalizing symptoms at nonfrontal sites, even though previous investigators have noted asymmetry differences in nonfrontal sites between depressed and nondepressed individuals. For example, several studies have observed greater relative left parietal activation in depressed individuals compared to controls (see Reid, Duke, \& Allen, 1998). Likewise, Bruder et al. (2005) found that adult offspring of depressed parents had greater relative left activation in medial posterior sites. Activation in right posterior sites has been involved in the processing of emotional stimuli (Moratti, Rubio, Campo, Keil, \& Ortiz, 2008), and thus hypoactivity in these sites, as reflected in greater relative left posterior activation, could result in low responsivity to positive as well as negative stimuli (Kovacs \& Lopez-Duran, 2010). However, it is possible that while asymmetry in posterior sites may contribute to depression by reducing affective range, such asymmetry may not be directly involved in the regulation of responses to stressful events. This is consistent with findings suggesting that frontal regions play a key role in modulating physiological responses to stress. The frontal cortex has direct reciprocal neural projections to the amygdala (Afifi \& Bergman, 1998). In animals, damage to the frontal cortex results in an overactivation of the amygdala, suggesting that the frontal cortex serves an inhibitory function on the stress response (e.g., Gewirtz, Falls, \& Davis, 1997; Morgan, Romanski, \& Ledoux, 1993). Likewise, individual variability in frontal but not posterior activation appears to modulate the hypothalamicpituitary-adrenal axis responses to stress (Buss et al., 2003; Kalin et al., 1998). Therefore, our results highlight the potential role of lateral frontal regions in mitigating the negative effects of exposure to high levels of stress in high-risk children.

The results of this paper should be considered in light of some limitations. First, internalizing symptoms, as well as life stressors, were reported by parents, which raises the possibility of informant bias and shared variance. However, we observed effects only for the internalizing but not for the externalizing problems (results not shown), which provides some support for the specificity and validity of these findings. In addition, recent investigations have shown the value and validity of parental report as compared to nonparental, third-party reports (see Kerr, Lunkenheimer, \& Olson, 2007). Second, each life stressor was coded as having or not having occurred, so that multiple occurrences of the same event had the same influence as a single occurrence of the event. This may have limited the differences between the samples in life stress exposure. Third, all variables were assessed concurrently, which limits our ability to conclusively establish the directionality of the results. For example, it is plausible that internalizing symptoms moderated the effect of life stress on asymmetry scores. Fourth, we did not have measures of actual affective responses to the films (e.g., facial expressions), and thus our hypothesized links to affective style are purely based on the observed EEG patterns. Finally, our sad film did not produce the expected asymmetry pattern in the low-risk group, or the expected change from baseline for both groups. This limits our ability to conclude whether the moderating effect of frontal asymmetry was unique to a specific affective style.

In conclusion, to our knowledge this is the first study to show that affective style, as indexed by frontal asymmetry, can moderate 
the effects of life stressors on the development of internalizing symptoms among children at high-risk for depression. We showed that a pattern of frontal EEG asymmetry associated with approachrelated motivation may dampen the harmful effects of stress exposure. The results contribute to our understanding of the mechanisms of risk among children at familial risk for depression and the role of brain electrical asymmetry in the responses to stressful events.

\section{References}

Achenbach, T. (1991). Manual for the Child Behavior Checklist/4-18 and the 1991 profile. Burlington,VT: University of Vermont.

Adrian, C., \& Hammen, C. (1993). Stress exposure and stress generation in children of depressed mothers. Journal of Consulting and Clinical Psychology, 61, 354-359.

Afifi, A., \& Bergman, R. (1998). Functional neuroanatomy. New York, NY: McGraw-Hill.

Allen, J. J. B., Coan, J. A., \& Nazarian, M. (2004). Issues and assumptions on the road from raw signals to metrics of frontal EEG asymmetry in emotion. Biological Psychology, 67, 183-218. doi: 10.1016/ j.biopsycho.2004.03.007

Allen, J. J. B., Harmon-Jones, E., \& Cavender, J. H. (2001). Manipulation of frontal EEG asymmetry through biofeedback alters self-reported emotional responses and facial EMG. Psychophysiology, 38, 685-693.

Alloy, L. B., Abramson, L. Y., Hogan, M. E., Whitehouse, W. G., Rose, D. T., Robinson, M. S., . . Lapkin, J. B. (2000). The Temple-Wisconsin Cognitive Vulnerability to Depression Project: Lifetime history of Axis I psychopathology in individuals at high and low cognitive risk for depression. Journal of Abnormal Psychology, 109, 403-418.

American Electroencephalographic Society. (1994). Guideline thirteen: Guidelines for standard electrode position nomenclature. Journal of Clinical Neurophysiology, 11, 111-113.

Ashman, S. B., Dawson, G., Panagiotides, H., Yamada, E., \& Wilkinson, C. W. (2002). Stress hormone levels of children of depressed mothers. Development and Psychopathology, 14, 333-349. doi: 10.1017/ S0954579402002080

Baron, R. M., \& Kenny, D. A. (1986). The moderator-mediator variable distinction in social psychological research: Conceptual, strategic, and statistical considerations. Journal of Personality and Social Psychology, 51, 1173-1182.

Bozdogan, H. (1987). Model selection and Akaike's information criterion (AIC): The general theory and its analytical extensions. Psychometrika, $52,345-370$.

Bruder, G. E., Tenke, C. E., Warner, V., Nomura, Y., Grillon, C., Hille, J., . . . Weissman, M. M. (2005). Electroencephalographic measures of regional hemispheric activity in offspring at risk for depressive disorders. Biological Psychiatry, 57, 328-335. doi: 10.1016/j.biopsych.2004. 11.015

Buchanan, T. W., \& Lovallo, W. R. (2001). Enhanced memory for emotional material following stress-level cortisol treatment in humans. Psychoneuroendocrinology, 26, 307-317. doi: 10.1016/S03064530(00)00058-5

Buss, K. A., Schumacher, J. R., Dolski, I., Kalin, N. H., Goldsmith, H. H., \& Davidson, R. J. (2003). Right frontal brain activity, cortisol, and withdrawal behavior in 6-month-old infants. Behavioral Neuroscience, $117,11-20$.

Caspi, A., Sugden, K., Moffitt, T. E., Taylor, A., Craig, I. W., Harrington, H., . . Poulton, R. (2003). Influence of life stress on depression: Moderation by a polymorphism in the 5-HTT gene. Science, 301, 386-389.

Coan, J. A., \& Allen, J. J. B. (2003). Frontal EEG asymmetry and the behavioral activation and inhibition systems. Psychophysiology, 40, 106-114.

Coan, J. A., \& Allen, J. J. B. (2004). Frontal EEG asymmetry as a moderator and mediator of emotion. Biological Psychology, 67, 7-50. doi: 10.1016/j.biopsycho.2004.03.002

Coan, J. A., Allen, J. J. B., \& McKnight, P. E. (2006). A capability model of individual differences in frontal EEG asymmetry. Biological Psychology, 72, 198-207. doi: 10.1016/j.biopsycho.2005.10.003

Davidson, R. J. (1993). Cerebral asymmetry and emotion: Conceptual and methodological conundrums. Cognition and Emotion, 7, 115-138.

Davidson, R. J. (1998). Affective style and affective disorders: Perspectives from affective neuroscience. Cognition \& Emotion, 12, 307. doi: $10.1080 / 026999398379628$

Davidson, R. J. (2004). What does the prefrontal cortex "do" in affect: Perspectives on frontal EEG asymmetry research. Biological Psychology, 67, 219-234. doi: 10.1016/j.biopsycho.2004.03.008
Davidson, R. J., \& Fox, N. A. (1982). Asymmetrical brain activity discriminates between positive and negative affective stimuli in human infants. Science, 218, 1235-1237.

Davidson, R. J., \& Fox, N. A. (1989). Frontal brain asymmetry predicts infants' response to maternal separation. Journal of Abnormal Psychology, 98, 127-131.

Davidson, R. J., Ekman, P., Saron, C. D., Senulis, J. A., \& Friesen, W. V. (1990). Approach-withdrawal and cerebral asymmetry: Emotional expression and brain physiology. I. Journal of Personality and Social Psychology, 58, 330-341.

Davidson, R. J., \& Irwin, W. (1999). The functional neuroanatomy of emotion and affective style. Trends in Cognitive Neurosciences, 3, $11-21$.

Davidson, R. J., Jackson, D. C., \& Kalin, N. H. (2000). Emotion, plasticity, context, and regulation: Perspectives from affective neuroscience. Psychological Bulletin, 126, 890-909.

Dawson, G., Frey, K., Panagiotides, H., Osterling, J., \& Hessl, D. (1997). Infants of depressed mothers exhibit atypical frontal brain activity: A replication and extension of previous findings. Journal of Child Psychology and Psychiatry, 38, 179-186. doi: 10.1111/j.1469-7610.1997. tb01852.x

Drevets, W. C. (2000). Neuroimaging studies of mood disorders. Biological Psychiatry, 48, 813-829. doi: 16/S0006-3223(00)01020-9

Field, T., Pickens, J., Fox, N. A., \& Nawrocki, T. (1995). Relative right frontal EEG activation in 3- to 6-month-old infants of "depressed" mothers: Special section: Parental depression and distress: Implications for development in infancy, childhood, and adolescence. Developmental Psychology, 31, 358-363.

Field, T., Pickens, J., Fox, N. A., Nawrocki, T., \& Gonzalez, J. (1995). Vagal tone in infants of depressed mothers. Development and Psychopathology, 7, 227-231. doi: 10.1017/S0954579400006465

Forbes, E. E., Fox, N. A., Cohn, J. F., Galles, S. F., \& Kovacs, M. (2006). Children's affect regulation during a disappointment: Psychophysiological responses and relation to parent history of depression. Biological Psychology, 71, 264-277. doi: 10.1016/j.biopsycho.2005.05.004

Forbes, E. E., Shaw, D. S., Fox, N. A., Cohn, J. F., Silk, J. S., \& Kovacs, M. (2006). Maternal depression, child frontal asymmetry, and child affective behavior as factors in child behavior problems. Journal of Child Psychology and Psychiatry, and Allied Disciplines, 47, 79-87. doi: 10.1111/j.1469-7610.2005.01442.x

Gasser, T., Bächer, P., \& Möcks, J. (1982). Transformations towards the normal distribution of broad band spectral parameters of the EEG. Electroencephalography and Clinical Neurophysiology, 53, 119-124. doi: 10.1016/0013-4694(82)90112-2

Gewirtz, J. C., Falls, W. A., \& Davis, M. (1997). Normal conditioned inhibition and extinction of freezing and fear-potentiated startle following electrolytic lesions of medical prefrontal cortex in rats. Behavioral Neuroscience, 111, 712-726.

Goodman, S. H., \& Gotlib, L. H. (1999). Risk for psychopathology in the children of depressed mothers: A developmental model for understanding mechanisms of transmission. Psychological Review, 106, 458-490.

Goodyer, I. M., Herbert, J., Tamplin, A., \& Altham, P. M. (2000). Recent life events, cortisol, dehydroepiandrosterone and the onset of major depression in high-risk adolescents. British Journal of Psychiatry, 177, 499-504.

Goodyer, I., Kolvin, I., \& Gatzanis, S. (1985). Recent undesirable life events and psychiatric disorder in childhood and adolescence. The British Journal of Psychiatry, 147, 517-523. doi: 10.1192/bjp.147.5.517

Jackson, D. C., Mueller, C. J., Dolski, I., Dalton, K. M., Nitschke, J. B., Urry, H. L., . . Davidson, R. (2003). Now you feel it, now you don't: Frontal brain electrical asymmetry and individual differences in emotion regulation. Psychological Science, 14, 612-617. doi: 10.1046/ j.0956-7976.2003.psci_1473.x

Jacobs, G. D., \& Snyder, D. (1996). Frontal brain asymmetry predicts affective style in men. Behavioral Neuroscience, 110, 3-6. doi: 10.1037/ 0735-7044.110.1.3 
Jones, N. A., Field, T., \& Almeida, A. (2009). Right frontal EEG asymmetry and behavioral inhibition in infants of depressed mothers. Infant Behavior and Development, 32, 298-304. doi: 10.1016/j.infbeh.2009. 04.004

Jones, N. A., Field, T., \& Davalos, M. (2000). Right frontal EEG asymmetry and lack of empathy in preschool children of depressed mothers. Child Psychiatry \& Human Development, 30, 189-204.

Jones, N. A., Field, T., Davalos, M., \& Pickens, J. (1997). EEG stability in infants/children of depressed mothers. Child Psychiatry \& Human Development, 28, 59-70.

Jones, N. A., Field, T., Fox, N. A., Lundy, B. L., \& Davalos, M. (1997). EEG activation in 1-month-old infants of depressed mothers. Development and Psychopathology, 9, 491-505.

Kalin, N. H., Larson, C., Shelton, S. E., \& Davidson, R. J. (1998). Asymmetric frontal brain activity, cortisol, and behavior associated with fearful temperament in rhesus monkeys. Behavioral Neuroscience, 112, 286-292.

Kerr, D. C. R., Lunkenheimer, E. S., \& Olson, S. L. (2007). Assessment of child problem behaviors by multiple informants: A longitudinal study from preschool to school entry. Journal of Child Psychology and Psychiatry and Allied Disciplines, 48, 967-975.

Ketter, T. A., George, M. S., Kimbrell, T. A., Benson, B. E., \& Post, R. M. (1996). Functional brain imaging, limbic function, and affective disorders. Neuroscientist, 2, 55-65.

Kovacs, M., \& Lopez-Duran, N. L. (2010). Prodromal symptoms and atypical affectivity as predictors of major depression in juveniles: Implications for prevention. Journal of Child Psychology and Psychiatry, 51, 472-496.

Lewis, R. S., Weekes, N. Y., \& Wang, T. H. (2007). The effect of a naturalistic stressor on frontal EEG asymmetry, stress, and health. Biological Psychology, 75, 239-247. doi: 10.1016/j.biopsycho.2007. 03.004

Lopez-Duran, N. L., Kovacs, M., \& George, C. J. (2009). Hypothalamic pituitary adrenal axis dysregulation in depressed children and adolescents: A meta-analysis. Psychoneuroendocrinology, 34, 1272-1283. doi: 10.1016/j.psyneuen.2009.03.016

Lopez-Duran, N. L., Vazquez, D. M., Felt, B., \& Olson, S. L. (2009). An integrative approach to the neurophysiology of emotion regulation. In S. L. Olson \& A. J. Sameroff (Eds.), Biopsychosocial regulatory processes in the development of childhood behavioral problems. New York, NY: Cambridge University Press.

Marshall, P. J., Bar-Haim, Y., \& Fox, N. A. (2002). Development of the EEG from 5 months to 4 years of age. Clinical Neurophysiology, 113, 1199-1208.

Mayer, L., Lopez-Duran, N. L., Kovacs, M., George, C. J., Baji, I., Kapornai, K., . . . Vetró, A. (2009). Stressful life events in a clinical sample of depressed children in Hungary. Journal of Affective Disorders, 115, 207-214. doi: 10.1016/j.jad.2008.08.018

Moratti, S., Rubio, G., Campo, P., Keil, A., \& Ortiz, T. (2008). Hypofunction of right temporoparietal cortex during emotional arousal in depression. Archives of General Psychiatry, 65, 532-541. doi: 10.1001/ archpsyc.65.5.532

Morgan, M. A., Romanski, L. M., \& Ledoux, J. E. (1993). Extinction of emotional learning: contribution of medial prefrontal cortex. Neuroscience Letters, 163, 109-113.

Niedermeyer, E. (1999). Maturation of the EEG: Development of waking and sleep patterns. In E. Niedermeyer \& F. Lopes da Silva (Eds.),
Electroencephalography: Basic principles, clinical applications, and related fields, (4th ed., pp. 189-214). Baltimore, MD: Williams \& Wilkins.

Oldfield, R. C. (1971). The assessment and analysis of handedness: The Edinburgh Inventory. Neuropsychologia, 9, 97-113.

Olino, T. M., Lopez-Duran, N. L., Kovacs, M., George, C. J., Gentzler, A. L., \& Shaw, D. S. (2011). Developmental trajectories of positive and negative affect in children at high and low familial risk for depressive disorder. Journal of Child Psychology and Psychiatry, 52, 792-799.

O'Reardon, J. P., Solvason, H. B., Janicak, P. G., Sampson, S., Isenberg, K. E., Nahas, Z., ... Sackeim, H. A. (2007). Efficacy and safety of transcranial magnetic stimulation in the acute treatment of major depression: A multisite randomized controlled trial. Biological Psychiatry, 62, 1208-1216. doi: 10.1016/j.biopsych.2007.01.018

Pan, W. (2001). Akaike's information criterion in generalized estimating equations. Biometrics, 57, 120-125.

Reeves, B., Lang, A., Thorson, E., \& Rothschild, M. (1989). Emotional television scenes and hemispheric specialization. Human Communication Research, 15, 493-508. doi: 10.1111/j.1468-2958.1989.tb00196.x

Reid, S. A., Duke, L. M., \& Allen, J. J. B. (1998). Resting frontal electroencephalographic asymmetry in depression: Inconsistencies Suggest the need to identify mediating factors. Psychophysiology, 35, 389-404. doi: 10.1017/S0048577298970986

Silk, J. S., Shaw, D. S., Skuban, E. M., Oland, A. A., \& Kovacs, M. (2006). Emotion regulation strategies in offspring of childhood-onset depressed mothers. Journal of Child Psychology and Psychiatry, 47, 69-78. doi: 10.1111/j.1469-7610.2005.01440.x

Smagin, G. N., Heinrichs, S. C., \& Dunn, A. J. (2001). The role of CRH in behavioral responses to stress. Peptides, 22, 713-724.

Thibodeau, R., Jorgensen, R. S., \& Kim, S. (2006). Depression, anxiety, and resting frontal EEG asymmetry: A meta-analytic review. Journal of Abnormal Psychology, 115, 715-729. doi: 10.1037/0021-843X.115.4. 715

Tomarken, A. J., Davidson, R. J., \& Henriques, J. B. (1990). Resting frontal brain asymmetry predicts affective responses to films. Journal of Personality and Social Psychology, 59, 791-801.

Tomarken, A. J., Dichter, G. S., Garber, J., \& Simien, C. (2004). Resting frontal brain activity: Linkages to maternal depression and socioeconomic status among adolescents. Biological Psychology, 67, 77-102.

Tops, M., Wijers, A. A., van Staveren, A. S. J., Bruin, K. J., den Boer, J. A., Meijman, T. F., \& Korf, J. (2005). Acute cortisol administration modulates EEG alpha asymmetry in volunteers: relevance to depression. Biological Psychology, 69, 181-193.

Vuga, M., Fox, N. A., Cohn, J. F., Kovacs, M., \& George, C. J. (2008). Long-term stability of electroencephalographic asymmetry and power in 3 to 9 year-old children. International Journal of Psychophysiology, 67, 70-77. doi: 10.1016/j.ijpsycho.2007.10.007

Wheeler, R. E., Davidson, R. J., \& Tomarken, A. J. (1993). Frontal brain asymmetry and emotional reactivity: A biological substrate of affective style. Psychophysiology, 30, 82-89.

Williamson, D. E., Birmaher, B., Frank, E., Anderson, B. P., Matty, M. K., \& Kupfer, D. J. (1998). Nature of life events and difficulties in depressed adolescents. Journal of the American Academy of Child \& Adolescent Psychiatry, 37, 1049-1057.

(RECEIVED March 11, 2011; ACCEPTED October 6, 2011) 